\title{
A waterways management framework for Western Australia
}

\author{
C. Macgregor, B. Stewart \& C. Farrell \\ Centre of Excellence in Natural Resource Management, \\ University of Western Australia, Australia
}

\begin{abstract}
The State of Western Australia (WA) occupies one third of the Australian continent. The hydro-climate varies enormously from the tropical monsoon region in the north; through an erratic, semi-arid climate of the northwest and interior; to temperate regions of the south. Most waterways in the State are intermittent, with summer flow in the north, winter flow in the south and ephemeral flows in the northwest. Perennial streams are comparatively rare geographically.

For much of WA there is insufficient water quality and riparian vegetation condition data to make comprehensive, objective assessments of the health of waterways. In many parts of the State, a comprehensive assessment of the values and condition of, and threats to, waterway ecosystems has not been undertaken. Most information and management is centred on the southwest land division and there is comparatively little known of waterways elsewhere. However, regional Natural Resource Management (NRM) groups and other organisations are mapping, classifying, evaluating and prioritising their waterway activities. This paper presents and describes the development of a State-wide waterways management framework. The framework is based on values-threats models and allows assessment of waterways' attributes even when comprehensive or inadequate data is unavailable. It is also capable of incorporating objective and subjective information so it could prove valuable to waterways managers operating in relatively remote regions where rapid appraisal-type assessments are required.
\end{abstract}

Keywords: waterways management framework, assets and threats assessment Western Australia. 


\section{Background}

Waterways (defined here as intermittent and/or perennial rivers, streams and wetlands) in Western Australia (WA) vary considerably. The north of WA is dominated by a tropical climate with dry 'winters' and wet 'summers' (monsoon). Central and interior parts of the State have an erratic, semi-arid climate with waterways flowing very intermittently. In contrast, the south of WA has a Mediterranean and temperate climate, but even here many waterways only run during winter.

For the majority of the State there is limited water quality and riparian vegetation condition data to make comprehensive, objective assessments of waterway health. In many parts of the State, a comprehensive assessment of the values of and threats to waterways' ecosystems has never been undertaken [1] and in other parts data is limited or inconsistent.

The State Government (Department of Water (DoW)) and regional Natural Resource Management (NRM) groups, have important roles in the management of the State's waterways. However, without a State-wide waterways management framework these organisations have had no objective, systematic method for prioritising management. This paper describes a waterways management prioritisation framework developed by the University of Western Australia [2] and now adopted by the State Government.

To be useful, the framework needed to support assessment of the values and condition of, and threats to, waterways even where data is limited or subjective. To ensure this, the following principles were adopted in the development of the framework:

- take cognisance of, and incorporate previous waterway assessment initiatives undertaken by government agencies and NRM groups in WA;

- be consistent with National waterway assessments, yet recognise the need to modify these to accommodate the wide variety of WA conditions;

- support the values-threats management approach adopted by the State;

- be designed to be applied at various scales, including at the waterway reach, sub-catchment and basin scale; and,

- have the capacity to be applied when only limited quantitative and/or and qualitative data is available.

As noted, the framework is based on the values-threats approach adopted by other NRM frameworks including; Department of Environment's Salinity Investment Framework [3] and the State-wide Waterways Needs Assessment model developed by Water and Rivers Commission [4].

\section{Values and threats criteria}

\subsection{Waterway values}

Values are socially constructed and even with waterways, there are no truly objective values that provide managers with clear decision-making information. However, the concept of sustainability offers a deterministic approach for 
considering values and also encourages holistic and integrated responses. Sustainability recognises three primary dimensions for setting out values: ecological, social and economic. Waterway values have been classified according to these three criteria (Table 1), with each suggested criterion requiring indicators and measures for assessment (detailed in the report by Macgregor et al. [2]).

Table 1: $\quad$ Values criteria for waterways management.

\begin{tabular}{|l|l|l|}
\hline \multicolumn{1}{|c|}{ Ecological values } & \multicolumn{1}{|c|}{ Social values } & \multicolumn{1}{c|}{ Economic values } \\
\hline - Naturalness/condition & - Visual amenity & - Water \& mineral \\
- Representativeness & - Recreational & extraction \\
- Diversity or richness & - Non-Indigenous & - Commercial \\
- Rarity & heritage & - Infrastructural \\
- Special features & - Educational & \\
& - Indigenous jurisdiction & \\
& - Spiritual & \\
& - Hunting/gathering & \\
\hline
\end{tabular}

\section{Threatening activities (causes)}

- Water development (e.g. infrastructure)
- Agriculture
- Sewerage treatment
- Fishing
- Land development (e.g. residential)
- Aquaculture
- In-stream barriers
- Boating
- Motorcycling
- 4WD
- Horse riding
- Camping
- Cycling
- Inappropriate access
- Water extraction
- Hunting/gathering

\section{Threatening processes (effects)}

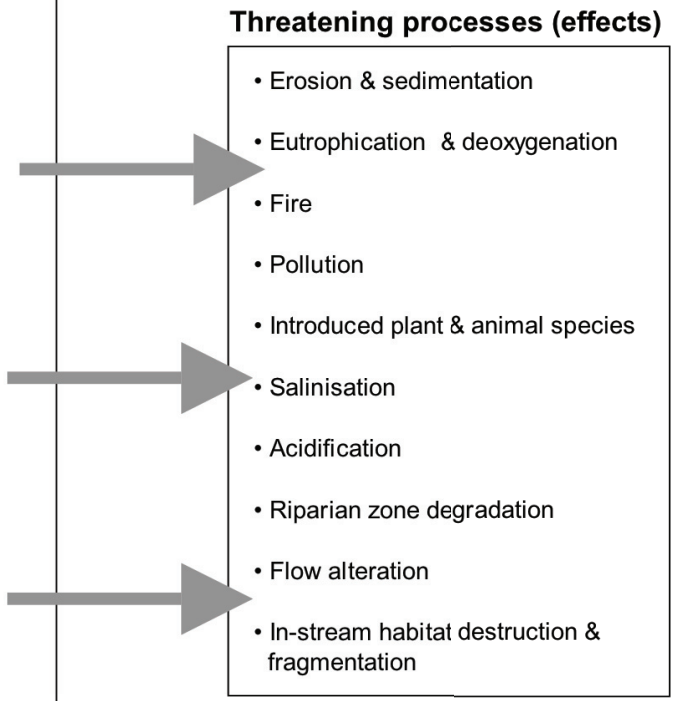

Figure 1: Threatening activities and processes impacting on waterway values. 


\subsection{Waterway threats}

Waterway threats are nearly always anthropomorphic, even if it is the environment's response to human activities that negatively impacts e.g. bank erosion and sedimentation as a result of excessive boating (Figure 1). Not all of these activities may be problematic however - it is only when they exceed ecological capacity that problems arise.

Some threatening activities are capable of causing many threatening processes while others are likely to cause one or two (e.g. inappropriate agriculture is capable of causing most of the threatening processes identified in Figure 1 whereas inappropriate cycling is likely to cause only minor erosion).

In a similar manner to values, threats can be considered under criteria, indicators and measures. While actual threats are associated with human activities (causes), the immediate management concern is likely to be processes (effects).

\section{Scoring and classification}

Some values and threats indicators will prove easier to score than others simply because it is easier to obtain data to represent them. Also, the quality and/or reliability of data may vary considerably. It will be important therefore to ensure that assessments are not driven by data. This means that scores generated for each indicator cannot simply be added together which would skew the result in favour of data-rich criteria. Each criterion should be scored against the same scale (e.g. 0 to 1.0) regardless of how well represented it is by the data.

\subsection{Data selection and reliability}

When considering values and threats it will be apparent that not all criteria apply in all circumstances. For example, if the waterway has no sewerage treatment plant then there will be no pollution threat of that kind. In short, assessments need only seek data to represent relevant criteria and indicators.

While data availability should not drive the scope of an assessment, inevitably, data availability will influence an assessment's reliability. Where there is 'good' data there will be a relatively high degree of confidence in the result. For all criteria that apply it will be beneficial to obtain as much data for as many of the measures and indicators as possible - to improve the reliability of the assessment. Where reliable quantitative data is not available, then qualitative and/or even subjective data will need to be utilised.

\subsection{Weighting}

While the sustainability model gives equal 'weight' to ecological, social and economic dimensions, it is likely that some criteria may be regarded more important than others in some circumstances. For example, a waterway reach containing a rare endemic species could be regarded as far more important than any social or economic values. In this situation 'weighting' could be required 
and while this may seem an inevitable and necessary element of the framework it is difficult to suggest a prescribed manner for applying weightings. It is likely that each case will have to be considered on its own merits, which suggests that stakeholder engagement should be included in the assessment process. That said, the basic approach for weighting could be as follows:

$$
W(\text { weighting })=x(\text { ecological })+y(\text { social })+z(\text { economic })=1.0
$$

For example, the case where there is presence of rare and threatened species:

$$
W=(x=0.75)+(y=0.2)+(z=0.05)
$$

\subsection{Classification}

Plotting river reaches, streams, or sub-catchments (depending on choice of scale) is a fairly straightforward process where the values and threats criteria combine to generate a single score for each. These are then plotted (Figure 2).

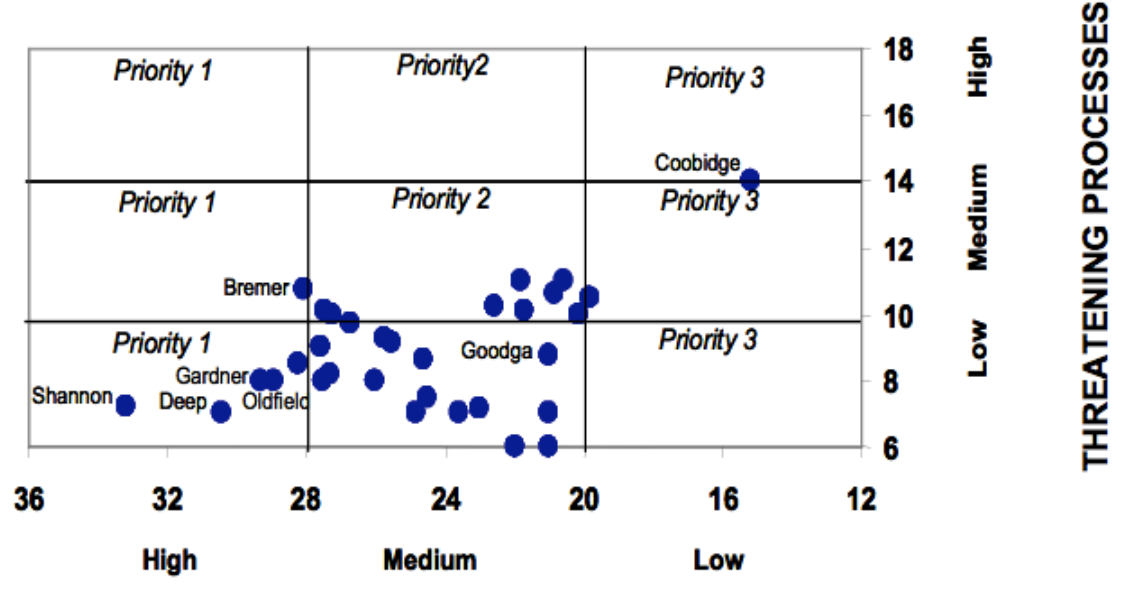

\section{ECOLOGICAL VALUES}

Figure 2: Summary matrix of values-threats for waterways in the South Coast region.

Once plotted, the reaches, streams or sub-catchments can then be overlaid into a simple $3 \times 3$ matrix (Figure 3). This figure demonstrates three primary priorities for management action: Priority 1, Priority 2 and Priority 3. Priority 1 reaches or sub-catchments should be considered the highest priority and consequently should receive attention for management first. Priority 2 reaches or waterways would receive attention second with those classed Priority 3 being considered last. In this framework waterway assets or values take precedence over threats. 


\begin{tabular}{|c|c|c|c|c|}
\hline & & \multicolumn{3}{|c|}{ VALUES } \\
\hline & & High & Medium & Low \\
\hline \multirow{3}{*}{ 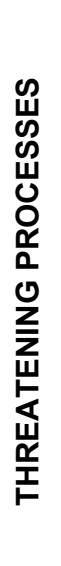 } & 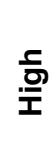 & $\begin{array}{c}\text { High value, } \\
\text { high threat } \\
\text { (HV/HT) } \\
\text { (Priority 1) }\end{array}$ & $\begin{array}{l}\text { Medium value, } \\
\text { high threat } \\
(\mathrm{MV} / \mathrm{HT}) \\
\text { (Priority 2) }\end{array}$ & $\begin{array}{c}\text { Low value, } \\
\text { high threat } \\
\text { (LV/HT) } \\
\text { (Priority 3) }\end{array}$ \\
\hline & $\frac{\xi}{\frac{5}{2}}$ & $\begin{array}{l}\text { High value, } \\
\text { medium threat } \\
(\text { HV/MT) } \\
(\text { Priority 1) }\end{array}$ & $\begin{array}{c}\text { Medium value, } \\
\text { medium threat } \\
\text { (MV/MT) } \\
\text { (Priority 2) }\end{array}$ & $\begin{array}{l}\text { Low value, } \\
\text { medium threat } \\
\text { (LV/MT) } \\
\text { (Priority 3) }\end{array}$ \\
\hline & 3 & $\begin{array}{c}\text { High value, low } \\
\text { threat } \\
\text { (HV/LT) } \\
\text { (Priority 1) }\end{array}$ & $\begin{array}{c}\text { Medium value, } \\
\text { low threat } \\
\text { (MV/LT) } \\
\text { (Priority 3) }\end{array}$ & $\begin{array}{c}\text { Low value, low } \\
\text { threat (LV/LT) } \\
\text { (Priority 3) }\end{array}$ \\
\hline
\end{tabular}

Figure 3: Values-threats prioritisation matrix.

The three-tier system of priorities suggested here is simplistic and should be sufficient in most circumstances; however, it does not consider how management should be prioritised within each of the three primary categories. For example, for Priority 1, there are three further categories or 'sub-priorities': high-value, high-threat (HV/HT); high-value, medium-threat (HV/MT); and, high-value, low-threat (HV/LT). Similarly, there are two sub-priorities for Priority 2 and four sub-priorities for Priority 3. By considering these sub-priority categories, further attention can be given to the institutional, social and economic constraints and limitations that are present is every NRM circumstance. In other words, the feasibility or practicality of management can be considered. For example, it is quite likely that management will be most effective if the less challenging threats are managed first.

\section{Management responses}

When it comes to management response options, the DoW [4] suggests six approaches. In this framework these have been altered slightly to include a seventh - monitoring - to reflect a more holistic management structure:

1. To fully protect waterways values:

- Secure: of such importance that action is needed to fully protect environmental, social and economic values.

- Maintain: prevent negative alteration to existing waterway condition, practices and standards. 
2. To improve waterway health:

- Restore: reinstate specific values, conditions, standards or practices.

3. To manage degradation:

- $\quad$ Stabilise: halt degradation processes.

- Contain: limit degradation processes.

4. To manage function:

- Adapt: accept that the waterway is highly degraded, identify the functions still operational and manage to those functions.

5. To identify drivers of change:

- Monitor: conduct regular assessments of water quality and riparian condition to identify emerging threats if and when they arise.

Table 2 links these response options with the prioritisation matrix.

Table 2: $\quad$ Generalised management responses.

\begin{tabular}{|c|c|c|}
\hline $\begin{array}{c}\text { Primary priority } \\
\text { level }\end{array}$ & Sub-priority level & $\begin{array}{c}\text { Dominant Management } \\
\text { Response/s }\end{array}$ \\
\hline 1 & 1a (HV/HT) & Secure; Stabilise; Restore \\
\hline 1 & $1 \mathrm{~b}(\mathrm{HV} / \mathrm{MT})$ & Secure; Maintain; Restore \\
\hline 1 & $1 \mathrm{c}(\mathrm{HV} / \mathrm{LT})$ & Monitor \\
\hline 2 & $2 \mathrm{a}(\mathrm{MV} / \mathrm{HT})$ & Stabilise; Contain; Restore \\
\hline 2 & $2 \mathrm{~b}(\mathrm{MV} / \mathrm{MT})$ & Contain \\
\hline 3 & $3 \mathrm{a}(\mathrm{MV} / \mathrm{LT})$ & Stabilise; Restore \\
\hline 3 & $3 \mathrm{~b}(\mathrm{LV} / \mathrm{HT})$ & Stabilise; Contain \\
\hline 3 & $3 \mathrm{c}(\mathrm{LV} / \mathrm{MT})$ & Contain \\
\hline 3 & $3 \mathrm{~d}(\mathrm{LV} / \mathrm{LT})$ & Adapt \\
\hline
\end{tabular}

\subsection{Priority 1}

High value waterways should be considered for management before waterways of medium or low value. This logic can also be found in other NRM contexts, for example the Bradley approach to prioritising weed management [5], Possingham's approach to protecting species [6], and more significantly, Pen's [7] and Rutherfurd's [8] approach to waterways management. All these authors acknowledge the pragmatic problems involved with NRM, especially costeffectiveness. For example, Rutherfurd et al. prioritise waterway management to ensure that the 'biggest bang for our buck' [8] is achieved; that is, management aims to decrease the majority of threats at the greatest rate per unit dollar (or time) invested. This reflects the first principle of Rutherfurd et al:: 'always preserve [or secure] rare waterways, or waterways that are in good condition, first' [8]. Consequently, management efforts within this priority are likely to be dominated by those that secure and/or stabilise waterways from threats. Where waterways are not exposed to threats or threats are minimal, only monitoring is necessary.

\subsubsection{Sub-priority 1a (HV/HT)}

Given the high-value, high-threat circumstance associated with waterways in this classification, it is likely that these waterways will require the greatest proportion 
of management - to protect and/or enhance high value assets - but high threats exposure also implies the greatest effort and resources. For example, if the waterway is exposed to grazing then fencing off should ensure stock do not threaten values. In situations where degradation is high it is also important to consider potential off-site impacts; whether degradation is or might be passed on to other high-value reaches currently unaffected - especially downstream e.g. bank erosion is likely to create a sedimentation problem downstream. Where this is a possibility then management efforts should first stabilise degradation. In most situations securing and maintaining assets will have priority over restoration, mainly because the cost of protection is typically about one-tenth the cost of restoration. Only after degradation has been stabilised can restoration strategies be considered, and again, management should seek to support valuable assets first e.g. the rare or endangered species, before attending to sections of less importance.

\subsubsection{Sub-priority $1 \mathrm{~b}(\mathrm{HV} / \mathrm{MT})$}

High-value assets in this sub-classification are exposed to threats that are slightly less significant than 1a. It is also likely that degrading processes are less of a threat to high-value stretches of waterways downstream. In that sense, there is not the sense of urgency apparent in 1a. Management should still secure assets where necessary, and although it is likely that some efforts will already have been made, on-going maintenance will be necessary. For example, fencing already in place should be maintained, weeds continually removed, and so on.

\subsubsection{Sub-priority 1c (HV/LT)}

Waterways that fall into this sub-category are perhaps slightly unique from the other two high-value sub-categories. Since these waterways are regarded as being in good condition and under little exposure from threats there is little need to actively manage - such waterways will 'manage themselves' so long as conditions remain stable, i.e. no new developments or degradation processes introduced. Therefore, if resources are available, managers can consider applying these to other high-value or even medium-value waterways to protect or improve their condition. However, this does not imply that waterways in this category can be ignored altogether; it would be prudent to consider establishing an appropriate longitudinal monitoring program.

\subsection{Priority 2}

Reaches or streams in this class will have been damaged by human impact to a greater or lesser degree, but there is potential to protect assets and recover less damaged waterways at reasonable cost. There are two sub-classifications within this priority with those classed as medium-value, medium-threat (2a) prioritised before medium-value, low-threat $(2 b)$. Once again, recognising relative costs of protection compared with restoration, the most widespread benefit will be obtained by stabilising existing degradation before considering restoration. Restoration should consider the forth principle of Rutherfurd et al. [8]: 'Improve the condition of reaches that are damaged, beginning with those that are easy to fix.' 


\subsubsection{Sub-priority 2a (MV/HT)}

Typically, the worse examples of waterways in this sub-category would be those that have been largely cleared of riparian and in-channel vegetation, with marginal water quality and some sediment deposition in the channel. Ideally, restoration is called for but as noted above, active degradation (erosion, weed spread and nutrient enrichment) should be stabilised or at least contained first. If restoration opportunities arise, pragmatism will dictate the order of attack. For example, it is easiest to expand a reach in relatively good condition, than to restore an isolated reach. Look for opportunities to enhance the higher values that are present. The following order of management should be considered:

1. Improve degraded assets in reaches with some high quality values.

2. Work on a poor quality reach that links two value-rich reaches.

3. Work on a poor quality reach connected by to a value-rich reach.

\subsubsection{Sub-priority $2 \mathrm{~b}(\mathrm{MV} / \mathrm{MT})$}

Management options for this and lower value waterways will inevitably be quite limited because higher value systems are likely to consume most of the available resources. Given the relatively high cost of restoration, efforts will have to be directed more towards containment of degradation processes. However, if restoration opportunities arise then the order of priorities identified for $2 \mathrm{a}$ apply.

\subsection{Priority 3}

Given the nature of waterways management, and heavy demands on resources, there will be very limited opportunity to manage Priority 3 waterways to bring about dramatic improvements in their condition. However, it is important to acknowledge that waterways in poor condition can be sources of threats to other more valuable waterways or reaches downstream. For example, Salvinia molesta, a highly invasive aquatic weed of national significance in Australia, can pose serious threats to reaches downstream. In such cases, management efforts must stabilise and contain degradation. For waterways that pose little or no threat, resources may be more usefully directed to protecting or restoring highervalue waterways - accepting that there is nothing that can effectively be done (adapt).

\subsubsection{Sub-priority 3a (MV/LT)}

This sub-category occupies a slightly unique place in this framework - it is the only medium-value category with a Priority 3 rating. This in itself suggests that these waterways should be considered for management before $3 b, 3 c$ and $3 \mathrm{~d}$, which are all lower value. However, there is perhaps another important reason and it again relates to the logic of Rutherfurd et al. [8]: 'fix the easy before the hard'. Management should largely be concerned with stabilising degradation with perhaps restoration where opportunities arise.

\subsubsection{Sub-priority 3b (LV/HT)}

Reaches and streams typical of this sub-category would be in very poor condition with little or no chance of recovery without significant restoration. 
Unfortunately restoration of these waterways will be expensive and/or difficult. In terms of management response, where highly degraded reaches have potential to threaten reaches downstream it is appropriate to take action to stabilise or contain. Where this is unlikely, there is a strong argument for doing nothing except protecting remaining ecosystem functions (adapt).

\subsubsection{Sub-priority 3c (LV/MT)}

Similar to $3 \mathrm{~b}$, management for reaches in this sub-category must mostly be concerned with containment; the nature of threats should not be as much of a concern as $3 \mathrm{~b}$ but since these are low-value systems there is likely little to be gained by investing heavily in active restoration.

\subsubsection{Sub-priority 3d (LV/LT)}

While waterways in this sub-category are of low-value they pose little if any threat to other higher value reaches. In terms of management there is probably less to do here than in any other sub-priority category. Adaptation is called for but remaining ecosystem functions could be managed to at least protect those. There is also a case for monitoring the waterway to ensure no significant changes.

\subsection{Management feasibility}

As indicated above, resources (finances, personnel, time) play a central role in all management. Like the framework proposed here, the SIF model [3] recognised this issue, which was generically described as 'feasibility'. This is in recognition that management responses to natural resource threats are constrained by the capacity of organisations, community or landholders to undertake required management. In terms of feasibility, DEC [3] suggested five questions relevant to waterways that should be considered:

- How much will the management response cost?

- How technically feasible is the management response?

- Will the management response effectively reduce the threat/s?

- How long will it take for the management response to reduce threat/s?

- Will the response be implemented/supported by relevant stakeholders?

Once a programme of management has been decided upon it is important to monitor progress and the performance indicators should be SMART (specific, measurable, achievable, relevant, timely).

\section{Stages involved in using the framework}

The first step involves identifying the purpose of assessment. This influences selection of values-threats criteria and indicators to be assessed, as well as the level of aggregation to be conducted. For example, broad-scale planning should involve ecological, social and economic values, and calculation of an overall value of a waterway management unit. However, if the purpose of were to protect biodiversity emphasis would be placed on ecological values. 
Step two involves choosing the scale of assessment e.g. reach, sub-catchment, or basin. This will largely depend on the geographic extent of the study and its context. Then, choose values and threats criteria to be used in the assessment. The report by Macgregor et al. [2] contains full details criteria, indicators and measures. Obtain data to represent the measures and if necessary include qualitative or subjective data for remote waterways where there is no readily available objective data.

The next step involves rating each waterway unit e.g. low, medium, high (1 3 ) for all relevant indicator measures. For each indicator, where more than one measure was used, add the rating scores for the individual measures and divide by the number of measures used to derive a mean rating for that indicator. Then, for each criterion, add indicator scores together to derive a total score for each criterion and from this plot values vs. threats for each waterway. This can then be over-laid into a suitably derived nine-box prioritisation matrix (Figure 2). Finally, to determine management actions, refer to the Generalised management responses table (Table 2 ).

\section{Conclusions}

The State-wide Waterways Management Framework has been developed to assist regional NRM groups and State government agencies classify, evaluate and prioritise waterway management projects. The framework is not intended to replace any existing prioritisation processes but rather to enhance what is already being done. However, where these types of decision-making processes are yet to be carried out, the framework is a relatively simple, quick, and yet fundamentally objective approach. The framework is also highly transparent enabling stakeholders to readily engage with the approach and provide input.

As part of the framework's development, four trials were carried out (detailed in the report by Macgregor et al. [2]). These trials demonstrated the framework effectively operates at different scales using a variety of data types. The trials also revealed the framework can be used by anyone with a moderate level of scientific expertise.

One of the challenges potential users of the framework will face is the question of values and threats data availability. This will particularly be the case for waterways in remote regions. Nevertheless, the framework is flexible enough to allow prioritisation assessments with very limited data using readily available web-based sources such as Google Earth and Australia's Wetlandbase.

\section{References}

[1] Department of Water, Catchment based waterways management framework for Western Australia: Classifying and evaluating waterways and prioritising management actions (unpublished working paper), 2004.

[2] Macgregor, C., Cook, B. \& Farrell, C., A Framework for the management of waterways in Western Australia, Report No CENRM070, Centre of Excellence in Natural Resource Management, University of Western 
Australia. Report (draft) prepared for the Department of Water, DoW ref: 0115-2007, 2008.

[3] Department of Environment, Salinity investment framework: interim report - phase 1, Department of Environment, Salinity and Land Use Impacts Series No. SLUI 32, Western Australia. Online. http://portal.water. wa.gov.au/portal/page/portal/WaterManagement/Salinity/SalinityInvestment Framework

[4] Water and Rivers Commission, State-wide waterways needs assessment: prioritising action for waterways management in Western Australia, WRC. Online. $\quad$ http://portal.water.wa.gov.au/pls/portal/url/page/waterways/ publications/technical_reports

[5] Bradley, J., Bringing back the bush: the Bradley method of bush regeneration, Landsdowne Press, Sydney, 1988.

[6] Possingham, H. P. Ecological Triage. Nature Australia, 27(8), 2002.

[7] Pen, L. J. Managing our rivers: a guide to the nature and management of the streams of south-west Western Australia, Water and Rivers Commission, East Perth, WA 6004, 1999.

[8] Rutherfurd, I., Jerie, K., Walker, M. \& Marsh, N., Don't raise the Titanic: How to set priorities for stream rehabilitation. Proc. of the Second Australian Stream Management Conference, Vol. 2, eds. I. Rutherfurd \& R. Bartley, Adelaide, pp. 527-532, 1999. 